\title{
INEQUALITIES FOR A COMPLEX MATRIX WHOSE REAL PART IS POSITIVE DEFINITE
}

\author{
BY
}

CHARLES R. JOHNSON $\left({ }^{1}\right)$

\begin{abstract}
Denote the real part of $A \in M_{n}(C)$ by $H(A)=1 / 2\left(A+A^{*}\right)$. We provide dual inequalities relating $H\left(A^{-1}\right)$ and $H(A)^{-1}$ and an identity between two functions of $A$ when $A$ satisfies $H(A)>0$. As an application we give an inequality (for matrices $A$ satisfying $H(A)>0$ ) which generalizes Hadamard's determinantal inequality for positive definite matrices.
\end{abstract}

0 . Introduction. Denote the real part of an $n$ by $n$ complex matrix $A$ by

$$
H(A) \equiv 1 / 2\left(A+A^{*}\right)
$$

and define $\Pi_{n}=\left\{A \in M_{n}(C): H(A)>0\right\}$. If $A \in \Pi_{n}$, then $A$ is nonsingular and $A^{-1} \in \Pi_{n}$. It is our goal to present inequalities relating the positive definite matrices $H\left(A^{-1}\right)$ and $H(A)^{-1}$ when $A \in \Pi_{n}$. These results may then be compared with different inequalities obtained in [4] for the same problem when $A$ is, in addition, restricted to have real entries. This leads to an identity linking two functions of $A$ when $A \in \Pi_{n}$. As an application of these inequalities we also present a result which generalizes Hadamard's determinantal inequality for positive definite matrices. We also generalize the Ostrowski-Taussky inequality for matrices in $\Pi_{n}$.

1. Main result. Proofs of the following useful fact may be found in [1] or [2].

Lemma. If $A \in \Pi_{n}$, then $A^{-1} A^{*}$ is similar to a unitary matrix.

In order to facilitate the statement of results, we define

$$
M=M(A) \equiv \max \operatorname{Re}(\lambda) \text { and } m=m(A) \equiv \min \operatorname{Re}(\lambda)
$$

where the maximum and minimum are taken over all eigenvalues $\lambda$ of $A^{-1} A^{*}, A$ nonsingular. In view of the lemma and the fact $I+A^{-1} A^{*}=A^{-1}\left(A+A^{*}\right)$ is invertible for $A \in \Pi_{n}$, we necessarily have (for $A \in \Pi_{n}$ ) that $-1<m(A) \leqslant M(A)$ $\leqslant 1$. Our main result is

Received by the editors July 2, 1974.

AMS (MOS) subject classifications (1970). Primary 15A09, 15A45, 15 A57.

Key words and phrases. Eigenvalues, Hadamard inequality, real part, positive definite.

( ${ }^{1}$ ) A portion of this work is included in the author's doctoral dissertation written under the advisement of Professor Olga Taussky Todd at the California Institute of Technology. 
THEOREM 1. If $A \in \Pi_{n}$, then

(i) $c H\left(A^{-1}\right)-H(A)^{-1}>0$ if and only if $c>2 /(m+1)$ (equivalently $m>$ $(2-c) / c)$, and

(ii) $d H(A)^{-1}-H\left(A^{-1}\right)>0$ if and only if $d>(M+1) / 2$ (equivalently $M<$ $2 d-1)$.

Proof. In the following calculation we let $\lambda(X)$ denote an arbitrary eigenvalue of the $n$ by $n$ matrix $X$.

$$
\begin{aligned}
& c H\left(A^{-1}\right)-H(A)^{-1}>0 \\
& \leftrightarrow \lambda\left(\left[c H\left(A^{-1}\right)\right]^{-1}\left(c H\left(A^{-1}\right)-H(A)^{-1}\right)\right)>0 \\
& \leftrightarrow \lambda\left(I-\left[c H(A) H\left(A^{-1}\right)\right]^{-1}\right)>0 \leftrightarrow \lambda\left(-\left[c H(A) H\left(A^{-1}\right)\right]^{-1}\right)>-1 \\
& \leftrightarrow \lambda\left(\left[c H(A) H\left(A^{-1}\right)\right]^{-1}\right)<1 \leftrightarrow \lambda\left(c H(A) H\left(A^{-1}\right)\right)>1 \\
& \leftrightarrow \lambda\left(H(A) H\left(A^{-1}\right)\right)>1 / c \leftrightarrow \lambda\left(\left(A+A^{*}\right) / 2 \cdot\left(A^{-1}+\left(A^{-1}\right)^{*}\right) / 2\right)>1 / c \\
& \text { (i) } \quad \leftrightarrow \lambda\left(I / 2+\left(A^{*} A^{-1}+A A^{-1 *}\right) / 4\right)>1 / c \\
& \leftrightarrow \lambda\left(\left(A^{*} A^{-1}+\left(A^{*} A^{-1}\right)^{-1}\right) / 4\right)>1 / c-1 / 2=(2-c) / 2 c \\
& \leftrightarrow \lambda\left(A^{*} A^{-1}+\left(A^{*} A^{-1}\right)^{-1}\right)>2((2-c) / c) \\
& \leftrightarrow \operatorname{Re} \lambda\left(A^{*} A^{-1}\right)=\operatorname{Re} \lambda\left(A^{-1} A^{*}\right)>(2-c) / c \\
& \leftrightarrow m>(2-c) / c \leftrightarrow c m>2-c \leftrightarrow c m+c>2 \\
& \leftrightarrow c(m+1)>2 \leftrightarrow c>2 /(m+1),
\end{aligned}
$$

and (i) is complete.

Note. Each matrix mentioned, excepting $A^{*} A^{-1}$ and $A^{-1} A^{*}$, has had necessarily real roots. In particular, since the roots of $A^{*} A^{-1}+\left(A^{*} A^{-1}\right)^{-1}$, or equivalently $A^{-1} A^{*}+\left(A^{-1} A^{*}\right)^{-1}$, are necessarily real, we have by this calculation alone that any complex roots of $A^{-1} A^{*}, A \in \Pi_{n}$, must be 1 in absolute value.

The proof of (ii) is similar.

(ii)

$$
\begin{aligned}
d H(A)^{-1} & -H\left(A^{-1}\right)>0 \\
& \leftrightarrow \lambda\left(\left[d H(A)^{-1}\right]^{-1}\left[d H(A)^{-1}-H\left(A^{-1}\right)\right]\right)>0 \\
& \leftrightarrow \lambda\left(I-H(A) H\left(A^{-1}\right) / d\right)>0 \leftrightarrow \lambda\left(-H(A) H\left(A^{-1}\right) / d\right)>-1 \\
& \leftrightarrow \lambda\left(-H(A) H\left(A^{-1}\right)\right)>-d \leftrightarrow \lambda\left(H(A) H\left(A^{-1}\right)\right)<d \\
& \leftrightarrow \lambda\left(I / 2+\left(A^{*} A^{-1}+\left(A^{*} A^{-1}\right)^{-1}\right) / 4\right)<d \\
& \leftrightarrow \lambda\left(A^{*} A^{-1}+\left(A^{*} A^{-1}\right)^{-1}\right)<(d-1 / 2) 4 \\
& \leftrightarrow \operatorname{Re} \lambda\left(A^{*} A^{-1}\right)=\operatorname{Re} \lambda\left(A^{-1} A^{*}\right)<(d-1 / 2) 2 \\
& \leftrightarrow M<2 d-1 \leftrightarrow M+1<2 d \leftrightarrow d>(M+1) / 2
\end{aligned}
$$

and the proof is complete. 
Corollary 1. $A \in \Pi_{n}$ implies $H(A)^{-1} \geqslant H\left(A^{-1}\right)$.

Proof. Since $M$ is at most 1 by the lemma, $d=1$ must satisfy $d \geqslant$ $(M+1) / 2$. The corollary then follows from part (ii) of Theorem 1 .

From Corollary 1 the following fact about determinants immediately follows:

Corollary 2. $A \in \Pi_{n}$ implies

$$
(\operatorname{det} H(A))^{-1} \geqslant \operatorname{det} H\left(A^{-1}\right) \text { and } \operatorname{det} H(A) H\left(A^{-1}\right) \leqslant 1 \text {. }
$$

2. Comparison to real case. We define

$$
S(A) \equiv 1 / 2\left(A-A^{*}\right) \text { and } T=T(A) \equiv \max _{j}\left\{\left|t_{j}\right|\right\}
$$

where $\pm i t_{j}$ are the eigenvalues of $H(A)^{-1} S(A)$, for $H(A)$ nonsingular. In [4] it is shown that

THEOREM 2. If $A \in \Pi_{n}, A$ is real, and $c$ is a real scalar, then $c H\left(A^{-1}\right)-$ $H(A)^{-1}>0$ if and only if $c>1+T^{2}$.

The validity of both Theorems 1 and 2 implies remarkably that

$$
m=\left(1-T^{2}\right) /\left(1+T^{2}\right) \text { or } T=((1-m) /(1+m))^{1 / 2}
$$

at least when $A \in \Pi_{n}$ has real entries. In fact this identity holds also when $A \in \Pi_{n}$ is complex.

THEOREM 3. For $A \in \Pi_{n}$,

$$
m=\left(1-T^{2}\right) /\left(1+T^{2}\right) \quad \text { or } \quad T=((1-m) /(1+m))^{1 / 2} .
$$

Proof. The two assertions

$$
m=\left(1-T^{2}\right) /\left(1+T^{2}\right) \text { and } T=((1-m) /(1+m))^{1 / 2}
$$

are equivalent. We shall prove the former. Again let $\lambda(X)$ denote an arbitrary eigenvalue of $X \in M_{n}(C)$.

First we obtain an expression for $T^{2}$, assuming $A \in \Pi_{n}$ :

$$
\begin{aligned}
T^{2} & =\max \left|\lambda\left(H(A)^{-1} S(A) H(A)^{-1} S(A)\right)\right|=\max \left(-\lambda\left(H(A)^{-1} S(A) H(A)^{-1} S(A)\right)\right) \\
& =-\min \left(\lambda\left(H(A)^{-1} S(A) H(A)^{-1} S(A)\right)\right) \\
& =-\min \left(\lambda\left(\left(A+A^{*}\right)^{-1}\left(A-A^{*}\right)\left(A+A^{*}\right)^{-1}\left(A-A^{*}\right)\right)\right) \\
& =-\min \left(\lambda\left(\left(I+A^{-1} A^{*}\right)^{-1}\left(I-A^{-1} A^{*}\right)\left(I+A^{-1} A^{*}\right)^{-1}\left(I-A^{-1} A^{*}\right)\right)\right) \\
& =-\min \left(\lambda\left(\left(I-A^{-1} A^{*}\right)^{2}\left(I+A^{-1} A^{*}\right)^{-2}\right)\right) .
\end{aligned}
$$

We therefore have

$$
\frac{1-T^{2}}{1+T^{2}}=\frac{1+\min \left(\lambda\left(\left(I-A^{-1} A^{*}\right)^{2}\left(I+A^{-1} A^{*}\right)^{-2}\right)\right)}{1-\min \left(\lambda\left(\left(I-A^{-1} A^{*}\right)^{2}\left(I+A^{-1} A^{*}\right)^{-2}\right)\right)}
$$

which we hope to show is equal to $m \equiv \min \operatorname{Re}\left(\lambda\left(A^{-1} A^{*}\right)\right)$. 
By the lemma of $\S 1$, the eigenvalues of $A^{-1} A^{*}$ are all of absolute value 1 . We have also noted that none of them is equal to -1 . If we let $\alpha_{1}, \cdots, \alpha_{n}$ be $n$ complex numbers of absolute value 1 , none of which is -1 , it then suffices to show that

$$
\min _{1 \leqslant j \leqslant n} \operatorname{Re} \alpha_{i}=\frac{1+\min _{1 \leqslant j \leqslant n}\left(\left(1-\alpha_{j}\right) /\left(1+\alpha_{j}\right)\right)^{2}}{1-\min _{1 \leqslant j \leqslant n}\left(\left(1-\alpha_{j}\right) /\left(1+\alpha_{j}\right)\right)^{2}} .
$$

Let $\alpha_{j}=a_{j}+i b_{j}, a_{j}^{2}+b_{j}^{2}=1, j=1, \ldots, n$. First note that $\left(\left(1-\alpha_{j}\right) /\left(1+\alpha_{j}\right)\right)^{2}$ $=-b_{j}^{2} /\left(1+a_{j}\right)$, a nonpositive real number, so that $\min _{1<j<n}\left(\left(1-\alpha_{j}\right) /\left(1+\alpha_{j}\right)\right)^{2}$ is well defined, nonpositive and is attained for some particular $\alpha_{j}$, call it $\alpha=a+b i$. Then the right-hand side of (*) evaluated at $\alpha$ is equal to

$$
\begin{aligned}
\frac{(1+\alpha)^{2}+(1-\alpha)^{2}}{(1+\alpha)^{2}-(1-\alpha)^{2}}=\frac{2+2 \alpha^{2}}{4 \alpha}=\frac{1+(a+b i)^{2}}{2(a+b i)} \\
=(1 /(a+b i)+a+b i) / 2=(a-b i+a+b i) / 2=a=\operatorname{Re}(\alpha) .
\end{aligned}
$$

Now, since $(1+t) /(1-t)$ is an increasing function of $t$ when $t \leqslant 0$ is real, it follows that the right-hand side of (*) is smallest (over all $\alpha_{j}, 1 \leqslant j \leqslant n$ ) when evaluated at $\alpha$, the minimizing value of $\left(\left(1-\alpha_{j}\right) /\left(1+\alpha_{j}\right)\right)^{2}$. Therefore the left-hand side of $(*)$ is also equal to $\operatorname{Re}(\alpha)$ and the proof is complete.

It is now clear that Theorem 2 is a corollary of Theorems 1 and 3 . In fact we may simply relax the assumption that $A$ is real in Theorem 2 .

Corollary 3. If $A \in \Pi_{n}$ and $c$ is a real scalar, then $c H\left(A^{-1}\right)-H(A)^{-1}>0$ if and only if $c>1+T^{2}$.

We also give another corollary which will be used later.

Corollary 4. For $A \in \Pi_{n}$, we have $m\left(A^{-1}\right)=m(A), M\left(A^{-1}\right)=M(A)$ and $T\left(A^{-1}\right)=T(A)$.

Proof. The first two asserted equalities follow from the lemma of $\S 1$ and the definitions of $m$ and $M$. The third follows from the fact that $T$ may be expressed as a function of $m$.

3. Hadamard generalization. For a positive definite hermitian matrix $A=\left(a_{i j}\right)$, Hadamard's inequality states that

$$
\operatorname{det} A \leqslant \prod_{i=1}^{n} a_{i i} \text {. }
$$

We shall say that a general matrix $A=\left(a_{i j}\right) \in M_{n}(C)$ satisfies the $H$-inequality if

$$
|\operatorname{det} A| \leqslant d(A) \equiv\left|\prod_{i=1}^{n} \operatorname{Re}\left(a_{i i}\right)\right| \text {. }
$$

Matrices in $\Pi_{n}$ do not necessarily satisfy the $H$-inequality, though, of course, the hermitian elements do. However, we may use Theorem 1 to obtain a generalization of Hadamard's inequality valid throughout $\Pi_{n}$. 
THeOREM 4. Suppose $A \in \Pi_{n}$. Then $|\operatorname{det} A| \leqslant k d(A)$ where $k=$ |det $c(I+B)^{-1} \mid, B=H(A)^{-1} S(A)$ and $c=1+T^{2}$.

Proof. Let $k=k(A)$ be as defined and we first note that $k\left(A^{-1}\right)=k(A)$. This is valid because of Corollary 4 and because

$$
\begin{aligned}
\left(\left(I+H\left(A^{-1}\right)^{-1} S\left(A^{-1}\right)\right)^{-1}\right)^{*} & =\left(A H\left(A^{-1}\right)\right)^{*}=A^{-1} H(A)=\left(H(A)^{-1} A\right)^{-1}, \\
\left(I+H(A)^{-1} S(A)\right)^{-i} & =(I+B)^{-1} .
\end{aligned}
$$

The fact that $A=H(A)[I+B]$ implies that

$$
\left|\operatorname{det} A^{-1}\right|=\left|\operatorname{det}(I+B)^{-1} \operatorname{det} H(A)^{-1}\right|
$$

which is $\leqslant\left|\operatorname{det}(I+B)^{-1} \operatorname{det} c H\left(A^{-1}\right)\right|$ because of Corollary 3. But.

$$
\left|\operatorname{det}(I+B)^{-1} \operatorname{det} c H\left(A^{-1}\right)\right|=\left|\operatorname{det} c(I+B)^{-1} \operatorname{det} H\left(A^{-1}\right)\right|=k \operatorname{det} H\left(A^{-1}\right)
$$

which is $\leqslant k d\left(A^{-1}\right)$ because of the original $H$-inequality. We thus have

$$
\left|\operatorname{det} A^{-1}\right| \leqslant k(A) d\left(A^{-1}\right) \text {. }
$$

However, because $k\left(A^{-1}\right)=k(A)$ and since $\Pi_{n}$ is closed under inversion, we may as well write $|\operatorname{det} A| \leqslant k(A) d(A)$.

REMARK 1. If $A \in \Pi_{n}$ is hermitian, then $k=1$ and we obtain the usual $H$-inequality as a special case.

REMARK 2. Because of Theorem 3 we may replace " $c=1+T^{2}$ " in Theorem 4 by " $c=2 /(m+1)$ ". Also, in case $A$ is real, the absolute value bars may be dropped throughout the previous argument.

REMARK 3. $0 \leqslant\left|\operatorname{det}(I+B)^{-1}\right| \leqslant 1 \leqslant c$ and $1 \leqslant\left|\operatorname{det} c(I+B)^{-1}\right|$ in Theorem 4.

ExAMPLE. Let $A=\left[\begin{array}{ll}2 & -1 \\ 1 & 1\end{array}\right]$. Then $A \in \Pi_{2}$ and equality is attained in the inequality asserted by Theorem 4 . In this case $\operatorname{det} A=3 ; d(A)=2 ; c=3 / 2$; $(I+B)^{-1}=(2 / 3)\left[\begin{array}{cc}1 & 1 / 2 \\ -1 & 1\end{array}\right]$ and $k=\operatorname{det} c(I+B)^{-1}=3 / 2$. Thus $\operatorname{det} A=3=$ $(3 / 2) 2=k d(A)$.

4. The Ostrowski-Taussky inequality. In [5] it is shown that for $A \in \Pi_{n}$ $\operatorname{det} H(A) \leqslant|\operatorname{det} A|$

and equality holds if and only if the skew-hermitian part $S(A)=0$. To some extent, the inequalities of this and other papers which have been cited are generalizations of the Ostrowski-Taussky inequality. We now give a direct generalization, the statement of which was suggested to us by M. Marcus.

THEOREM 5. If $A \in \Pi_{n}$, then

$$
|\operatorname{det} A|^{2 / n} \geqslant(\operatorname{det} H(A))^{2 / n}+|\operatorname{det} S(A)|^{2 / n} \text {. }
$$


Equality holds if and only if each eigenvalue of $H(A)^{-1} S(A)$ has the same absolute value.

Proof. It suffices to assume $H(A)=I$ because since each component is positive

$$
|\operatorname{det} A|^{2 / n} \geqslant(\operatorname{det} H(A))^{2 / n}+|\operatorname{det} S(A)|^{2 / n}
$$

if and only if

$$
\begin{aligned}
& (\operatorname{det} H(A))^{-1 / n}|\operatorname{det} A|^{2 / n}(\operatorname{det} H(A))^{-1 / n} \\
& \quad \geqslant(\operatorname{det} H(A))^{-1 / n}\left[\left(\operatorname{det}^{-} H(A)\right)^{2 / n}+|(\operatorname{det} S(A))|^{2 / n}\right](\operatorname{det} H(A))^{1 / n}
\end{aligned}
$$

or equivalently

$$
|\operatorname{det}(I+S)|^{2 / n} \geqslant(\operatorname{det} I)^{2 / n}+|\operatorname{det} S|^{2 / n}
$$

where $S$ is the skew-hermitian matrix $H(A)^{-1 / 2} S(A) H(A)^{-1 / 2}$.

Now let $t_{1}, \ldots, t_{n}$ be real numbers such that the eigenvalues of $S$ are $i t_{1}$, $\ldots, i t_{n}$. Then $|\operatorname{det}(I+S)|^{2}=\Pi_{j=1}^{n}\left(1+t_{j}^{2}\right)$ and we thus must show that

$$
\prod_{j=1}^{n}\left(1+a_{j}\right)^{1 / n} \geqslant 1+\left(\prod_{j=1}^{n} a_{j}\right)^{1 / n}
$$

where the $a_{j}=t_{j}^{2}$ are arbitrary nonnegative numbers, $j=1, \ldots, n$. But this latter statement is just Minkowski's well-known inequality in which equality is attained if and only if $a_{1}=a_{2}=\ldots=a_{n}$. Since the eigenvalues of $S$ are the same as those of $H(A)^{-1} S(A)$, this completes the proof of the theorem.

As a corollary it follows that another term may be added linearly to the Ostrowski-Taussky result.

Corollary 5. If $A \in \Pi_{n}$, then

$$
|\operatorname{det} A| \geqslant \operatorname{det} H(A)+|\operatorname{det} S(A)| \text {. }
$$

\section{REFERENCES}

1. C. R. DePrima and C. R. Johnson, The range of $A^{-1} A^{*}$ in $G L(n, C)$, Linear Algebra Appl. 9 (1974), 209-222.

2. Ky Fan, Generalized Cayley transforms and strictly dissipative matrices, Linear Algebra Appl. 5 (1972), 155-172. MR 45 \#5145.

3. - On real matrices with positive definite symmetric component, Linear Multilinear Algebra 1 (1973), $1-4$.

4. C. R. Johnson, An inequality for matrices whose symmetric part is positive definite, Linear Algebra Appl. 6 (1973), 13-18. MR 47 \#251.

5. A. M. Ostrowski and $O$. Taussky, On the variation of the determinant of a positive definite matrix, Nederl. Akad. Wetensch. Proc. Ser. A 54 = Indag. Math. 13 (1951), 383385. MR 13, 900.

INSTITUTE FOR FLUID DYNAMICS AND APPLIED MATHEMATICS, UNIVERSITY OF MARYLAND, COLLEGE PARK, MARYLAND 20742 